\title{
Buffering reproductive science in the era of COVID-19
}

\section{David F. Albertini ${ }^{1}$}

Published online: 18 May 2020

(C) Springer Science+Business Media, LLC, part of Springer Nature 2020

Never before has the interface between medicine and society been so blurred as what we are witnessing today. After coming to a standstill for many private clinics practicing human ARTs, and undergoing a major reassignment of duties for ART practitioners working in academic settings, the COVID-19 pandemic has suddenly altered the dimension of reproductive medicine on a global scale. And in the broader public health arena, the litany of facts, fallacies, and formidable fantasies about the science underlying the pathobiology of SARS-CoV2, the Covid-19 crisis has secured its place in the history of our planet as the trigger for all things political in the (mis)management of international health. Understanding its etiology, promulgating protective measures aimed at limiting the spread of disease, and ushering in testing, screening, and hopes for a vaccine have all conspired to place in our midst a landscape like no other before it. And where the practice of reproductive medicine will ultimately arrive is anyone's guess at this stage.

Fortunately, the world's leading organizations in our specialty have responded acutely and consistently as the pandemic continues to pose an evolving threat to our current state of wellbeing. And given the need to collect data from the molecular to epidemiological levels, our woefully inadequate knowledge base can only stand to improve with passing time. The current ambiguous diagnoses and treatments for the disease are understandably founded in the paucity of information available about this virus, its mode of infection at the cellular level, and the urgent need to absolve it from having anything to do with reproductive health. ASRM, ESHRE, IFFS and others are providing the measured and thoughtful guidance needed for getting back to business in as safe a set of conditions as is possible.

How JARG will serve our readership in the COVID-19 pandemic remains to be determined but from past experience, sorting fact from fallacy, and real science from inuendo are the building blocks upon which a cogent and practical hold on all matters ART will be scrutinized and delivered with utmost integrity.

How does the COVID-19 crisis impact standards of ART care before, during or after the situation resolves? Significant changes in practice are being contemplated making the world of ARTs a different place for future recipients of our skills and talents. Screening of gamete donors for infectious disease will be expanded as will whatever the world of research brings to the forefront as infected individuals join the ranks of patients seeking gametes or embryos for their own procreative purposes. And one thing above all else we have learned about the causes of infertility and subfertility since the dawning of contemporary reproductive medicine and biology is that fecundity is the first thing that suffers when a living organism undergoes challenges of this magnitude to health.

Here at JARG we will continue to educate our readership as ARTs and reproductive genetics foster a deeper understanding of human reproduction and the ways the COVID-19 crisis may influence stakeholders and practitioners alike. The precarious link between science and society is again being challenged and we purveyors of global reproductive health for all must continue to protect future generations in the post COVID-19 era.

David F. Albertini

eicjarg@gmail.com

1 Center for Human Reproduction, New York, NY, USA 